EPJ Web of Conferences 13, 06005 (2011)

DOI: $10.1051 /$ epjconf/20111306005

(C) Owned by the authors, published by EDP Sciences, 2011

\title{
Off-shell transport of broad resonances and dilepton production at SIS energies
}

\author{
Gy. Wolf ${ }^{1, a}$, B. Kämpfer ${ }^{2}$, and M. Zétényi ${ }^{1}$ \\ 1 KFKI RMKI, H-1525 Budapest, POB 49, Hungary \\ 2 Forschungszentrum Dresden-Rossendorf, Institut für Strahlenphysik, PF 510119, 01314 Dresden, Germany
}

\begin{abstract}
The time evolution of vector meson spectral functions is studied within a Boltzmann-UehlingUhlenbeck (BUU) type transport model. Applications focus on $\rho$ and $\omega$ mesons being important pieces for the interpretation of the dielectron invariant mass spectrum. Since the evolution of the spectral functions is driven by the local density, the in-medium modifications turn out to compete, in this approach, with the known vacuum contributions.
\end{abstract}

\section{Introduction}

Dielectrons serve as direct probes of dense nuclear matter stages during the course of heavy-ion collisions. The superposition of various sources, however, requires a deconvolution of the spectra by means of models. Of essential interest are the contributions of the light vector mesons $\rho$ and $\omega$. The spectral functions of both mesons are expected to be modified in a strongly interacting environment. Measurements with HADES [1,2] explore systematically the dilepton production at beam energies in the few $\mathrm{AGeV}$ region.

In our transport model of the BUU type the time evolution of single-particle distribution functions of various hadrons is evaluated within the framework of a kinetic theory. The $\rho$ meson is already a broad resonance in vacuum, while the $\omega$ meson may acquire a noticeable width in nuclear matter [3]. Therefore, one has to propagate properly the spectral functions of the $\rho$ and $\omega$ mesons. This is the main goal of our paper. Similar investigations were carried out by Bratkovskaya and Cassing in [4].

\section{Off-shell transport of broad resonances}

Recently, theoretical progress has been made in describing the in-medium properties of particles starting from the Kadanoff-Baym equations for the Green functions of particles. Applying a first-order gradient expansion after a Wigner transformation $[5,6]$ one arrives at a transport equation for the retarded Green function. In the medium, particles acquire a selfenergy $\Sigma(x, p)$ which depends on position and momentum as well as the local properties of the surrounding medium. Particle properties are described by

\footnotetext{
a e-mail: wolf@rmki.kfki.hu
}

their spectral function being the imaginary part of the retarded propagator

$$
\begin{aligned}
& \mathcal{A}(p)=-2 \operatorname{Im} G^{r e t}(x, p) \\
= & \frac{\hat{\Gamma}(x, p)}{\left(E^{2}-\mathbf{p}^{2}-m_{0}^{2}-\operatorname{Re} \sum^{r e t}(x, p)\right)^{2}+\frac{1}{4} \hat{\Gamma}(x, p)^{2}},
\end{aligned}
$$

where the resonance widths $\Gamma$ and $\hat{\Gamma}$ are related via the $\hat{\Gamma}(x, p)=-2 \operatorname{Im} \Sigma^{r e t} \approx 2 m_{0} \Gamma$, and $m_{0}$ is the vacuum pole mass of the respective particle.

To solve numerically the Kadanoff-Baym equations one may exploit the test-particle ansatz for the retarded Green function $[5,6]$. This function can be interpreted as a product of particle number density multiplied with the spectral function $\mathcal{A}$.

The relativistic version of the equation of motion have been derived in ref. [5]:

$$
\begin{aligned}
\frac{d \mathbf{x}}{d t}= & \frac{1}{1-C} \frac{1}{2 E} \\
& \left(2 \mathbf{p}+\partial_{p} \operatorname{Re} \Sigma^{r e t}+\frac{m^{2}-m_{0}^{2}-\operatorname{Re} \Sigma^{r e t}}{\hat{\Gamma}} \partial_{p} \hat{\Gamma}\right) \\
\frac{d \mathbf{p}}{d t}= & \frac{-1}{1-C} \frac{1}{2 E}\left(\partial_{x} \operatorname{Re} \Sigma^{r e t}+\frac{m^{2}-m_{0}^{2}-\operatorname{Re} \Sigma^{r e t}}{\hat{\Gamma}} \partial_{x} \hat{\Gamma}\right), \\
\frac{d E}{d t}= & \frac{1}{1-C} \frac{1}{2 E}\left(\partial_{t} \operatorname{Re} \Sigma^{r e t}+\frac{m^{2}-m_{0}^{2}-\operatorname{Re} \Sigma^{r e t}}{\hat{\Gamma}} \partial_{t} \hat{\Gamma}\right)
\end{aligned}
$$

with the renormalization factor

$$
C=\frac{1}{2 E}\left(\partial_{E} \operatorname{Re} \Sigma^{r e t}+\frac{m_{n}^{2}-m_{0}^{2}-\operatorname{Re} \Sigma^{r e t}}{\hat{\Gamma}} \partial_{E} \hat{\Gamma}\right)
$$

In the above, $m=\sqrt{E^{2}-\mathbf{p}^{2}}$ is the mass of an individual test-particle. The selfenergy $\Sigma^{r e t}$ is considered to be a function of density $n$, energy $\mathrm{E}$, and momentum $\mathbf{p}$. 
The change of the test-particle mass $m$ can be more clearly seen combining Eqs. (4) and (5) to

$$
\frac{d m^{2}}{d t}=\frac{1}{1-C}\left(\frac{d}{d t} \operatorname{Re} \Sigma^{r e t}+\frac{m^{2}-m_{0}^{2}-\operatorname{Re} \Sigma^{r e t}}{\hat{\Gamma}} \frac{d}{d t} \hat{\Gamma}\right)
$$

with the comoving derivative $d / d t \equiv \partial_{t}+\mathbf{p} / E \partial_{x}$. This equation means that the square of the particle mass tends to reach a value shifted by the real part of the selfenergy within a range of the value of $\hat{\Gamma}$. Thus, the vacuum spectral function is recovered when the particle leaves the medium.

The equations of motion of the test-particles have to be supplemented by a collision term which couples the equations of the different particle species. It can be shown [6] that the collision term has the same form as in the standard BUU treatment.

The in-medium spectral functions of $\omega$ and $\rho$ mesons also have to be taken into account when their test-particles are created. In the resonance decay the mass distribution of the generated test-particles for mesons results from an interplay of phase-space effects and the in-medium spectral functions $\mathcal{A}$ of the created meson. For the decay of a resonance of mass $m_{R}$ in a meson of mass $m$ and a baryon of mass $m_{N}$ we use the phase space distribution in the final state with a constant matrix element squared $|\mathcal{M}|^{2}$

$$
\Gamma=\mathcal{N} \int d^{4} p_{N} \delta\left(p_{N}^{2}-m^{2}\right) \int d^{4} p_{V} \frac{1}{2 \pi} \mathcal{A}\left(p_{V}\right)|\mathcal{M}|^{2}
$$

from which the distribution

$$
\frac{d N^{R \rightarrow N V}}{d m_{V}}=\mathcal{N} m_{V} \lambda^{1 / 2}\left(m_{R}^{2}, m_{N}^{2}, m_{V}^{2}\right) \mathcal{A}\left(m_{V}\right)
$$

results, where $\lambda$ is the triangle function $\lambda\left(a^{2}, b^{2}, c^{2}\right)=\left(c^{2}-\right.$ $\left.a^{2}-b^{2}\right)^{2}-(2 a b)^{2} . \mathcal{N}$ is an appropriate normalization factor.

We also include meson production during a transition $R \rightarrow R^{\prime} V$ from a resonance state $R$ to another resonance $R^{\prime}$ with $R^{\prime}=\Delta(1232), N(1440), N(1520), N(1535)$.

\subsection{Selfenergies}

To solve the Eqs. (3-5) one needs the knowledge of the selfenergies. Here one faces the need to decide which effects to take into account in the expression for the retarded selfenergy $\Sigma^{r e t}$ in the medium. That is because the BUU transport equations themselves already contain some part of in-medium effects that usually are considered in theoretical models in local density and local equilibrium approximation [7-9]. For instance, models for in-medium effects of $\rho$ mesons usually take into account the $N(1520)-$ nucleon-hole loop for the selfenergy, the corresponding vertices are accounted for in BUU via $\rho$-nucleon scattering and absorption through the $N(1520)$ resonance.

In our calculations we employ a simple form of the selfenergy of a vector meson $V$ :

$$
\begin{aligned}
& \operatorname{Re} \sum_{V}^{r e t}=2 m_{V} \Delta m_{V} \frac{n}{n_{0}} \\
& \operatorname{Im} \Sigma_{V}^{r e t}=m_{V}\left(\Gamma_{V}^{v a c}+\frac{n v \sigma_{V}}{\sqrt{1-v^{2}}}\right) .
\end{aligned}
$$

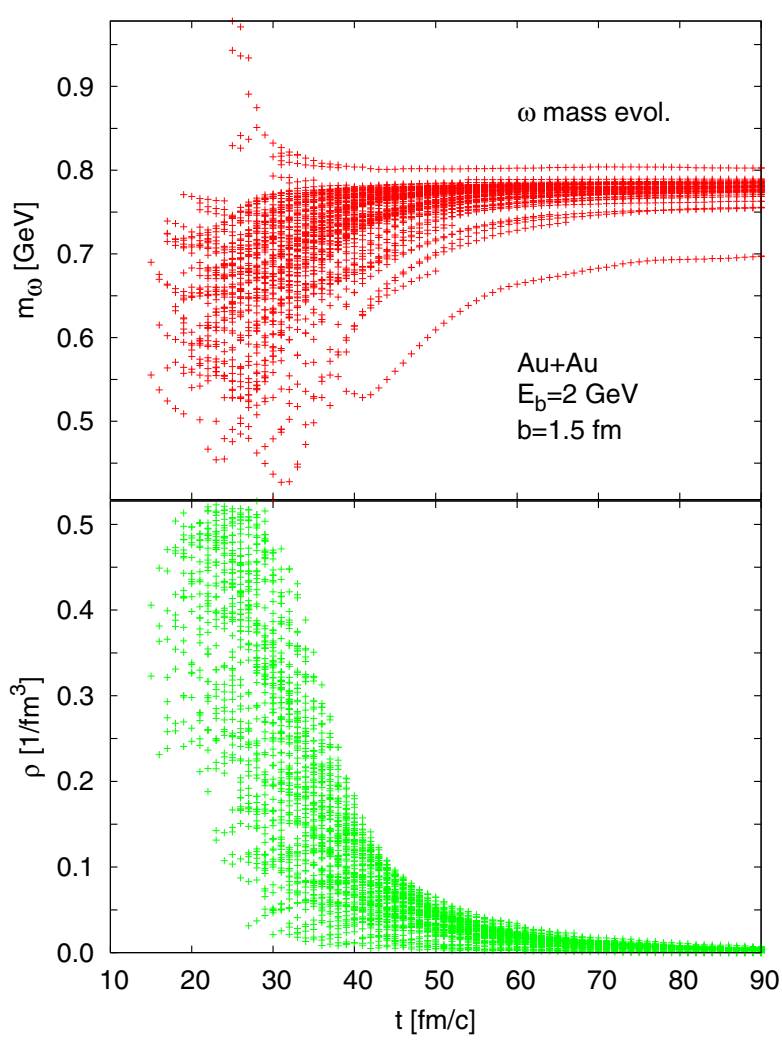

Fig. 1. Time evolution of the "masses" of several test-particles of $\omega$ mesons in a central $\mathrm{Au}+\mathrm{Au}$ collision at $2 \mathrm{AGeV}$ kinetic beam energy for an impact parameter of $1.5 \mathrm{fm}$ (upper panel). In the lower panel we show the corresponding densities experienced by the $\omega$ 's.

Eq. (10) describes a "mass shift" $\Delta m=\sqrt{m_{V}^{2}+\operatorname{Re} \sum_{V}^{r e t}}-$ $m_{V}$ characterized by $\Delta m_{V}$ and roughly being proportional to the density of the surrounding matter. The imaginary part contains the vacuum width $\Gamma_{V}^{v a c}$. The second term in Eq. (11) results from the collision broadening which depends on density $n$, relative velocity $v$ and the cross section $\sigma_{V}$ of the vector meson in matter. This cross section $\sigma_{V}$ is calculated via the Breit-Wigner formula

$$
\sigma_{V}=\frac{4 \pi}{q_{i n}^{2}} \sum_{R} \frac{2 J_{R}+1}{3\left(2 J_{i}+1\right)} \frac{s \Gamma_{V, R} \Gamma_{R}^{t o t}}{\left(s-m_{R}^{2}\right)^{2}+s\left(\Gamma_{R}^{t o t}\right)^{2}}
$$

for forming resonances with masses $m_{R}$, angular momenta $J_{R}$, partial widths $\Gamma_{V, R}$, total widths $\Gamma_{R}^{\text {tot }}$ with energy $\sqrt{s}$ and relative momentum $q_{\text {in }}$ in the entrance channel. In vacuum the baryon density $n$ vanishes and the resulting spectral function $\mathcal{A}_{v a c}$ is solely determined by the energy dependent width $\Gamma_{V}^{v a c}$. We remark that the life time of a testparticle is determined partially by its vacuum width. The life time is, furthermore, reduced by the absorption during the two-particles colisions which characterizes the total width $\operatorname{Im} \Sigma_{V}^{r e t}$.

If a $\rho$ meson is generated at normal density its mass is distributed in accordance with the spectral function. If 

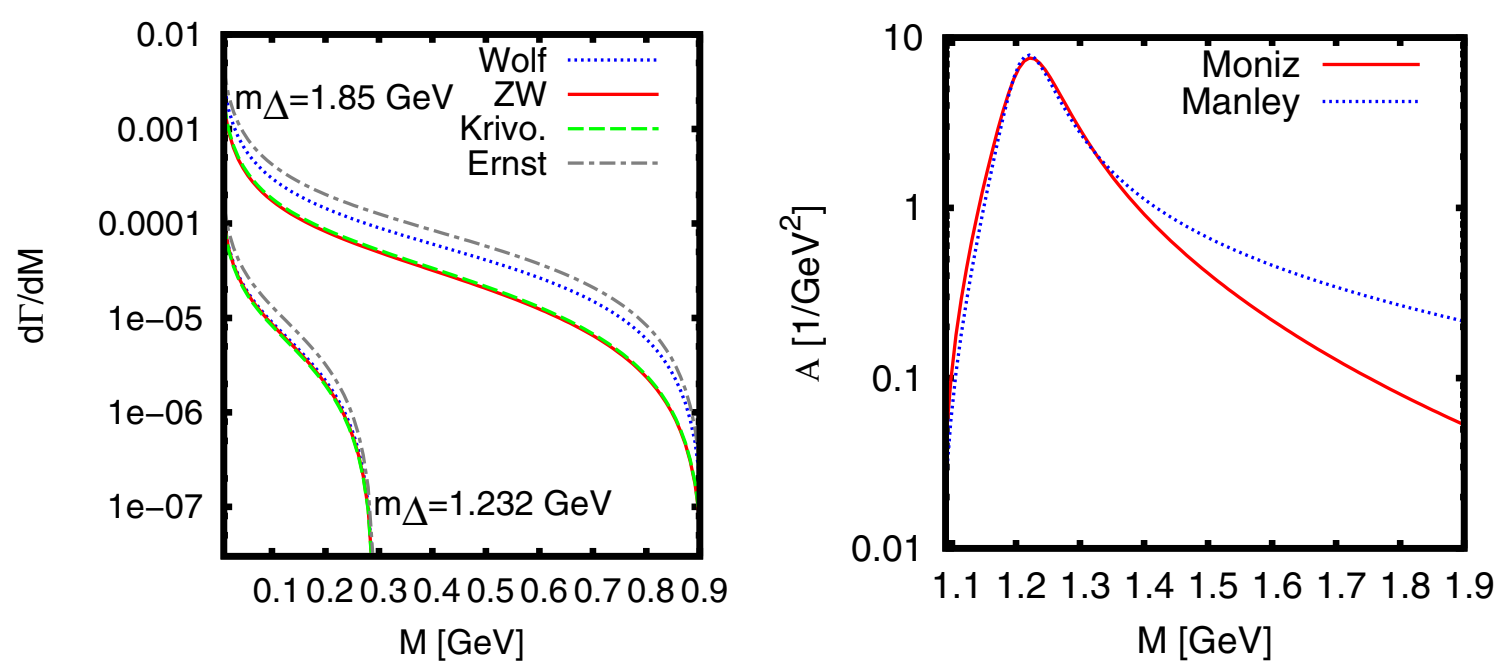

Fig. 2. Dalitz-decay of the $\Delta(1232)$ resonance for two masses: $1.232 \mathrm{GeV}$ and $1.85 \mathrm{GeV}$ where the descriptions from above $[10,13$, $15,16]$ (left panel) and the spectral function with two different cut-off prescriptions (right panel).

the meson propagates into a region of higher density then the mass will be lowered according to the action of $\operatorname{Re} \Sigma^{\text {ret }}$ in Eq. (7). However if the meson comes near the threshold the width $\hat{\Gamma}$ becomes very small and the second term of the right hand side of Eq. (7) dominates, i.e. reverses this trend leading to an increase of the mass.

In Fig. 1 we show how the masses of test-particles representing $\omega$ mesons are developing in heavy-ion collisions. At the end of the collision process, where the density is very low, the masses reach the vacuum value.

\section{Dielectron production}

The dielectrons stem from various sources; a detailed description can be found in refs. [10-12]. Direct vector meson decays $V \rightarrow e^{+} e^{-}$are dealt with integrating the local decay probabilities along their trajectories. Important contributions come from bremsstrahlung, Dalitz decays of the $\pi^{0}, \eta$ and $\omega$ mesons and the excited baryonic resonances as well emitting a dielectron together with a photon [13].

The bremsstrahlung contribution and the Dality decay of baryon resonances are presently not yet fully settled [12]. We include into our simulations a bremsstrahlung contribution which is guided by a one-boson exchange model adjusted to $p p$ virtual bremsstrahlung and transferred to $p n$ virtual bremsstrahlung [14].

The Dalitz decay of the baryonic resonances is as in ref. [13]. The most important contribution to the dielectron spectra of these come from the $\Delta(1232)$ resonance. There are other models $[16,10,15]$ for that Dalitz decay. As on the left panel of Fig. 2 can be seen these models agree very well for resonance decays from the peak mass, however they differ substantially for $\Delta$ resonances with masses relevant for studying the vector meson region. There is another uncertainty concerning these high-mass $\Delta(1232)$ resonances. The width and consequently the spectrum of these resonances are sensitive on the cut-off for high masses.
Here we show two possible paramerization: one from Moniz [17] and the other one from Manley used in the Particle Data Book [18].

The number of $\Delta(1232)$ resonances at around $1.85 \mathrm{GeV}$ masses may depend on the cut-off prescription by a factor of 3. Their Dalitz decay, as one can see in the left panel may differ by a factor of 4 . So the the Dalitz-decay contribution of the $\Delta(1232)$ resonance is uncertain by more than an order of magnitude in the vector meson region. This uncertainty may only be clarified by a detailed comparison of the calculation for $p p \rightarrow p p e^{+} e^{-}$with experimental data, using the angular dependence to localize the different channels in the data and then fix their magnitude. Here we would like to mention that different groups use different prescriptions for that channel, therefore, it is one important reason why the predictions especially the $\Delta(1232)$ Dalitz decay contributions are different.

\subsection{Results}

We investigate the distribution of the emitted dielectrons from $\omega$ mesons as a function of the density of the emitting region in Fig. 3. We consider three different density regions: (i) the density $n<n_{0} / 3$, (ii) $n_{0} / 3<n<n_{0}$, and (iii) $n>n_{0}$. For the light $C+C$ system dielectrons from all region have similar masses and can therefore hardly disentangled in experiment. For the $A u+A u$ system, dielectrons from the dense region have low masses around $600 \mathrm{MeV}$ and contribute roughly $10 \%$ to the total $\omega$ yield.

In Fig. 4 we exhibit dielectron spectra for $\mathrm{C}(2 \mathrm{AGeV})$ $+\mathrm{C}$. For a comparison with the HADES data the HADES filter has been applied [19] accounting for the geometrical acceptance, momentum cuts and pair kinematics. The filter causes a reduction of the strength and a smearing of the invariant masses of the dielectrons. In the left panel we show calculations where mass shifts and collisional broadening were applied for $\omega$ and $\rho$ mesons. In the figure we show 

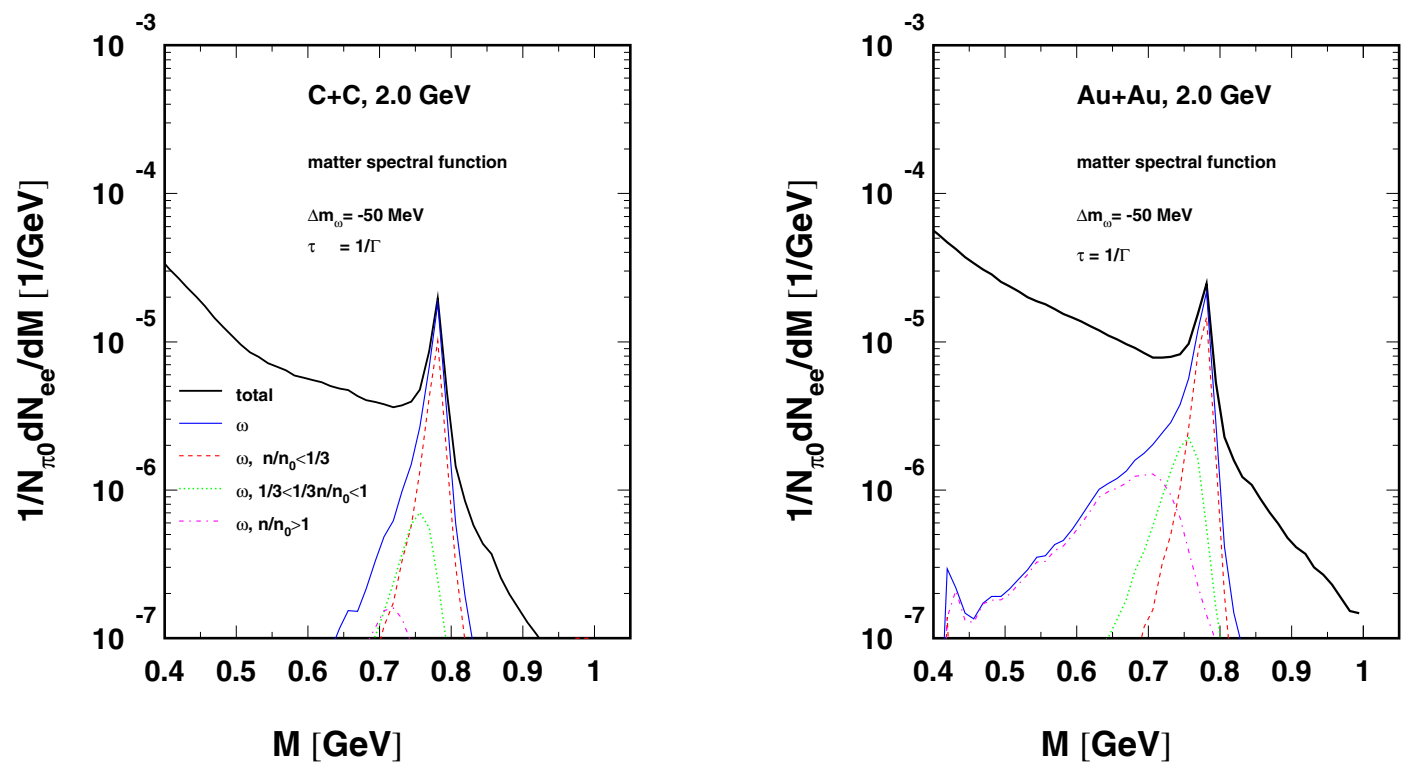

Fig. 3. Contribution to the dielectron yield from $\omega$ 's in various density regions compared with the total yield (thick solid line labelled by 'total'). The left picture is calculated for light $(\mathrm{C}+\mathrm{C})$ and the right one for heavy $(\mathrm{Au}+\mathrm{Au})$ systems.

various contributions to the dielectron rate. Important lowmass dielectron sources are $\pi^{0}$ and $\eta$ Dalitz decays which are proportional to the multiplicities of their parents. The TAPS collaboration has measured [20] the $\pi^{0}$ and $\eta$ production cross sections of $707 \pm 72 \mathrm{mb}$ and $25 \pm 4 \mathrm{mb}$ which have to be compared to our calculations of $870 \mathrm{mb}$ and 23 $\mathrm{mb}$ in the same reaction at the same energy. While the values for pion production are overestimated, the $\eta$ production is quite in agreement with the data. (Note that the presently employed cross sections rely on a global fit of many elementary reactions which is not optimized individually for the $\eta$ channel.) The Dalitz decays of $\rho$ and $\omega$ mesons and nucleon resonances do not contribute noticeably.

The overall agreement is very good. The mass shifts and broadening of the vector mesons do not have a considerable effect on the overall shape of the di-electron spectra although the peak position of the $\rho$ mesons is clearly shifted from 0.7 to $0.5 \mathrm{GeV}$. However, the large contributions of the cocktail of the other sources masks the effect of the $\rho$ mesons. Furthermore, most of the $\omega$ mesons decay outside the dense zone and are therefore not sensitive for medium effects. Since the fine structure ( $\omega$ peak) is not yet resolved in the data a conclusive decision cannot be made.

Progress could be made if the dielectron mass resolution is improved to identify the $\omega$ peak. However, our calculations do not point to the possibility of a two-peak structure (resulting from a superposition of vacuum decays and in-medium decays) or a substantial smearing of the $\omega$ peak due to a density dependent shift.

The present numerical set up provides a reasonable description of the HADES data [1] for dielectron masses below $0.6 \mathrm{GeV}$. In the higher mass region some overestima- tion of the data is recognized. On the right panel we compare the results with vacuum spectral function (solid curve) the dotted curves employ in-medium $\rho$ and $\omega$ spectral functions. The difference is small giving little hope to observe these medium effects on the vector mesons in the dilepton spectra for light systems. However, for heavy systems (Fig. 5 ) the difference is much larger, giving a reasonable chance for observing it.

We acknowledge the continuousily provided information by the HADES collaboration. The work is supported by the BMBF 06DR9059, GSI and OTKA T71989.

\section{References}

1. G. Agakichiev et al. (HADES collaboration), Phys. Rev. Lett. 98 (2007) 052302.

2. G. Agakichiev et al. (HADES collaboration), Phys. Lett. B663 (2008) 43.

3. Gy. Wolf, B. Friman, M. Soyer, Nucl. Phys. A640 (1998) 129.

4. E.Bratkovskaya and W.Cassing, Nucl. Phys. A807 (2008) 214.

5. W. Cassing, S. Juchem, Nucl. Phys. A672 (2000) 417.

6. S. Leupold, Nucl. Phys. A672 (2000) 475.

7. M. Lutz, Gy. Wolf, B. Friman, Nucl Phys. A706 (2002) 431.

8. W. Peters, M. Post, H. Lenske, S. Leupold, U. Mosel, Nucl. Phys. A632 (1998) 109.

9. M. Post, S. Leupold, U. Mosel, Nucl. Phys. A689 (2001) 753. 

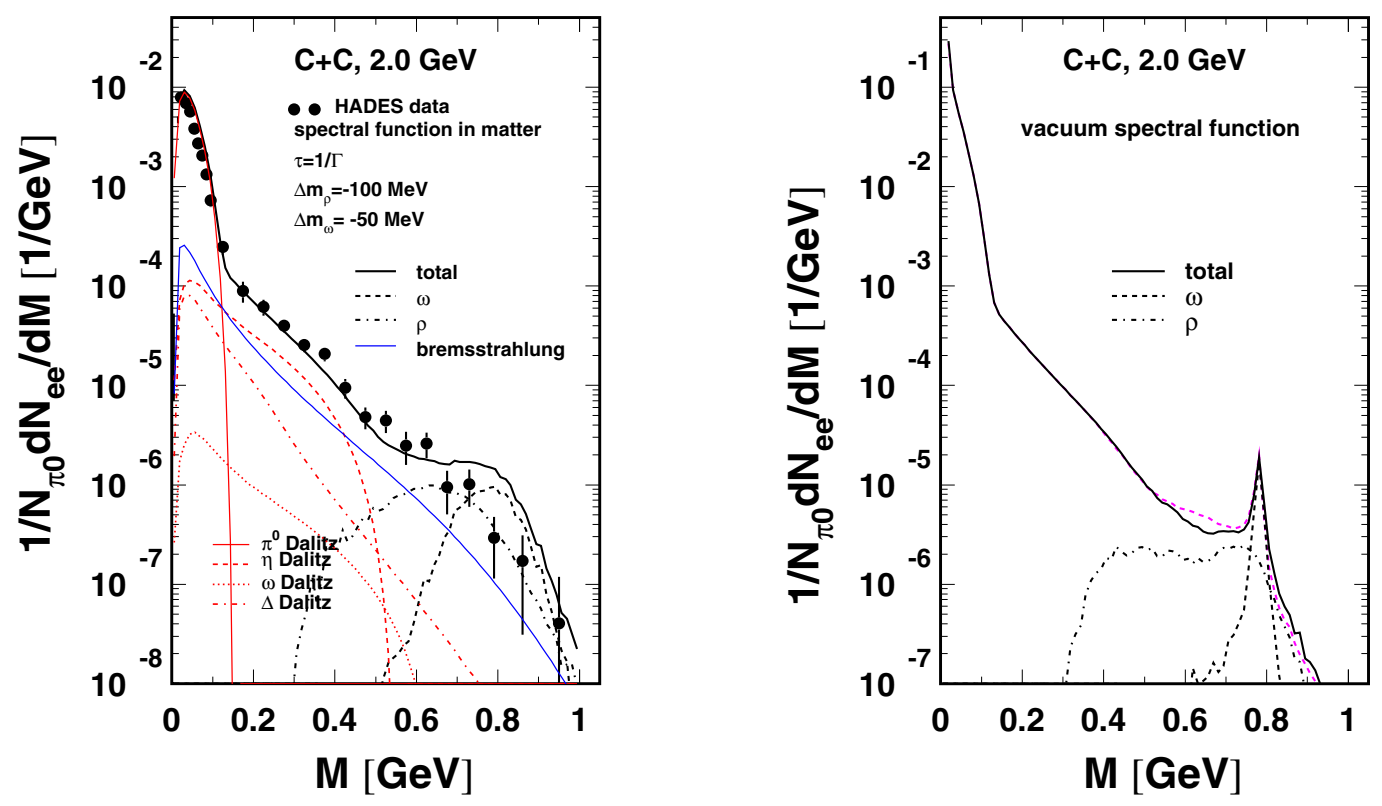

Fig. 4. Dielectron invariant mass spectra for $\mathrm{C}(2 \mathrm{AGeV})+\mathrm{C}$ collisions calculated with the in-medium spectral functions for parameters exhibited in the legend. The various sources of the dielectron invariant mass spectrum are indicated. The left panel shows the spectra after applying the experimental filter compared to HADES data [1]. The right panel compares the results for vacuum spectral functions (solid curve for the total yield) and in-medium $\rho$ and $\omega$ spectral functions (dashed curves).

10. Gy. Wolf, G. Batko, W. Cassing, U. Mosel, K. Niita, and M. Schäfer, Nucl. Phys. A517 (1990) 615.

11. Gy. Wolf, W. Cassing, U. Mosel, Nucl. Phys. A552 (1993) 549;

S. Teis, W. Cassing, M. Effenberger, A. Hombach, U. Mosel, G. Wolf, Z. Phys. A356 (1997) 421.

12. H.W. Barz, B. Kampfer, Gy. Wolf, M. Zetenyi, The Open Nuclear and Particle Physics Journal 3 (2010) 1.

13. M. Zétényi, Gy. Wolf, Phys. Rev. C67 (2003) 044002.

14. L. Kaptari, B. Kämpfer, Nucl. Phys. A764 (2006) 338.

15. M. I. Krivoruchenko, B. V. Martemyanov, A. Faessler and C. Fuchs, Annals Phys. 296 (2002) 299.

16. C. Ernst et al., Phys. Rev. C58 (1998) 447.

17. J.H. Koch, E.J. Moniz and N. Ohtsuka, Ann. Phys. 154 (1984) 99

18. D. E. Groom et al., The European Physical Journal C15 (2000) 1.

19. R. Holzmann et al. (HADES collaboration).

20. R. Averbeck et al. (TAPS collaboration), Z. Phys. A359 (1997) 65.

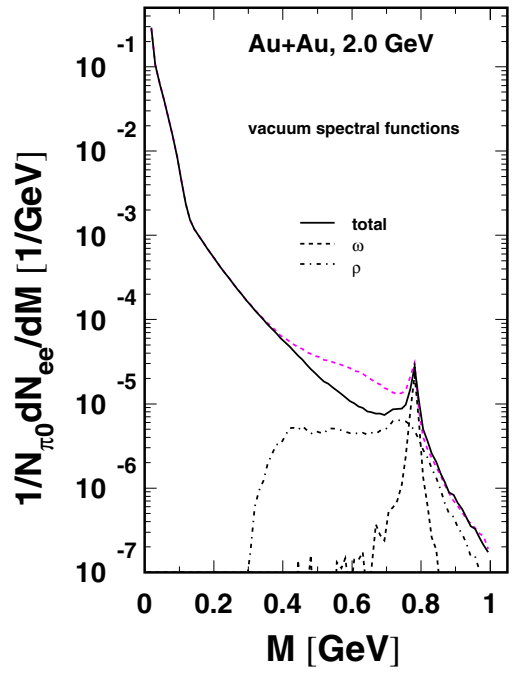

Fig. 5. Dielectron invariant mass spectra for $\mathrm{Au}(2 \mathrm{AGeV})+\mathrm{Au}$ collisions. Compared are the results for vacuum spectral functions (solid curve for the total yield) and in-medium $\rho$ and $\omega$ spectral functions (dashed curves). 